(C2008 IEEE. Personal use of this material is permitted. However, permission to reprint/republish this material for advertising or promotional purposes or for creating new collective works for resale or redistribution to servers or lists, or to reuse any copyrighted component of this work in other works must be obtained from the IEEE 


\title{
Application of Wavelet Modulus Maxima in Microarray Spots Recognition
}

\author{
X. H. Wang, Robert S. H. Istepanian, Senior Member, IEEE, and Yong Hua Song, Senior Member, IEEE
}

\begin{abstract}
This paper presents a novel approach to recognize the microarray image spots. The approach is based on the detection of wavelet modulus maxima in the microarray images. The detected maxima is actually the contour of the spots and thus the spots are recognized precisely. Then, the intensities within the contour of the spots can be obtained with low error rate. The test results on example image show this is an effective approach, especially for those spots with low intensities.
\end{abstract}

Index Terms-Wavelet analysis, microarray, images processing denoising.

\section{INTRODUCTION}

$\mathbf{I}$ N A MICROARRAY experiment, thousands of DNA sequences are printed in a high-density array on a solid surface. These sequences are referred to spot in image processing. Ideally, the spot on a microarray has the shape of a circle with a diameter consistent with all the other spots of that microarray. However, in practical experiments, not only the size of the spots is different from each other, but the shape of the spot is irregular. Some spots have the artefacts because of the experimental treatment, while others have low density to make them very difficult to be detected.

Spot recognition in microarray image processing is a crucial step to accurately obtain the gene expression. Over the last few years, many image processing methods have been adapted toward the spots recognition. These methods can be classified into three categories, namely, circle, adaptive shape, and histogram method. The fixed circle method with a constant diameter is simple to use and implemented in most of the common commercial software packages, such as ScanAlyze [1], GenePix [2], and QuantArray [3]. GenePix also offer the ability to allow the user to manually adjust the circle diameter spot by spot. This is time consuming. The drawback of this kind of method is evident. It is only accurate for regular round-shaped spots with exactly the same diameter as the circle. The adaptive shape method overcomes the drawbacks of the circle method. The two most commonly used are watershed [4], [5] and seeded region growing

\footnotetext{
Manuscript received January 15, 2003; revised June 16, 2003. Asterisk indicates corresponding author.

*X. H. Wang is with the Mobile Information and Network Technologies Centre, School of Computing and Information Systems, Kingston University, London Kingston Upon Thames KT1 2EE, U.K. (e-mail: xinheng.wang@kingston.ac.uk).

R. S. H. Istepanian is with Mobile Information and Network Technologies Centre, School of Computing and Information Systems, Kingston University, London, Kingston Upon Thames KT1 2EE, U.K.

Y. H. Song is with the Department of Electronic and Computer Engineering, Brunel University, Uxbridge UB8 3PH, U.K.

Digital Object Identifier 10.1109/TNB.2003.816230
}

[6], [7] methods. These two methods require the specification of starting points, or seeds, and the weak point of the methods can be the selection of the number and location of the seed point. However, in terms of microarray images, the number and the location of the spots are determined. These methods prove to be very effective.

The histogram method uses a target mask which is chosen to be larger than any spot. For each spot, the intensity is determined in some fashion from the histogram of pixel values within the masked area. One of the methods is using circular target mask [8]. However, a major disadvantage is that quantitation is unstable when a large target mask is set to compensate for spot size variation.

In this paper, a new approach is presented to accurately recognize the spots. The method is based on detection of wavelet transform modulus maxima. It is s well known that wavelet transform can localize the signal in space and frequency and, thus, easily characterize the local regularity of functions. In images, that would be the locations of the object contours. From locating the contours, the object can be recognized. If it is in the microarray image processing, that is the spot recognition by locating the edges of the spot. Actually, this is an edge-based spot recognition method. Because of the ability of the wavelet transform, this method is particularly useful in recognizing the low-intensity spots.

\section{Modulus Maxima in Image Edge Detection}

Modulus maxima is defined by Mallat and Zhong in their algorithm to detect the singularity at any point $\left(s_{0}, x_{0}\right)$, such that $\partial W f\left(s_{0}, x\right) / \partial x$ has a zero-crossing at $x_{0}$ and for any neighborhood of $x_{0}\left|W f\left(s_{0}, x\right)\right|<\left|W f\left(s_{0}, x_{0}\right)\right|$, where $W f(s, x)$ is the wavelet transform of real function $f(x)$ [9]. The local maxima are detected only along a dyadic sequence of scales to obtain efficient numerical implementations. For the algorithm to detect the local maxima in [9], two wavelets are proposed by partially derivatives of a two-dimensional (2-D) smoothing function $\theta(x, y)$ along $x$ and $y$

$$
\psi^{1}(x, y)=\frac{\partial \theta(x, y)}{\partial x} \quad \text { and } \quad \psi^{2}(x, y)=\frac{\partial \theta(x, y)}{\partial y} .
$$

Let $\psi_{s}^{1}(x, y)=(1 / s)^{2} \psi^{1}(x / s, y / s)$ and $\psi_{s}^{2}(x, y)=$ $(1 / s)^{2} \psi^{2}(x / s, y / s)$. The wavelet transform for function $f(x, y)$ with respect of $\psi^{1}(x, y)$ and $\psi^{2}(x, y)$ are obtained as

$$
\begin{aligned}
& W^{1} f(s, x, y)=f * \psi_{s}^{1}(x, y) \text { and } \\
& W^{2} f(s, x, y)=f * \psi_{s}^{2}(x, y) .
\end{aligned}
$$




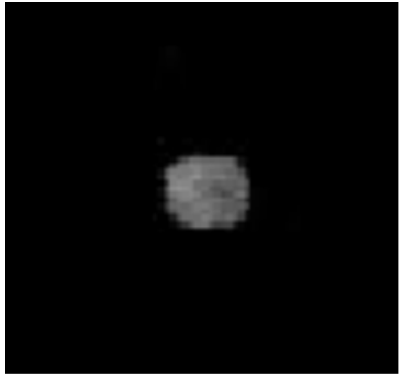

(a)

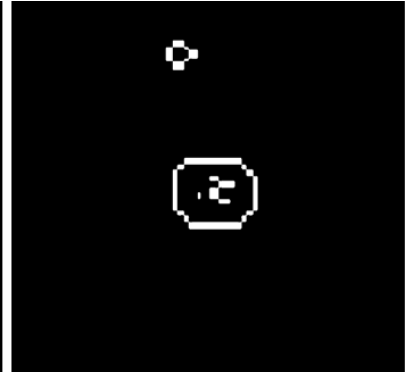

(b)
Fig. 1. Microarray spot and spot contour.

For a dyadic sequence, these become to $W^{1} f\left(2^{j}, x, y\right)$ and $W^{2} f\left(2^{j}, x, y\right)$. The value of each of these is bounded by

$$
M f\left(2^{j}, x, y\right)=\sqrt{\left|W^{1} f\left(2^{j}, x, y\right)\right|^{2}+\left|W^{2} f\left(2^{j}, x, y\right)\right|^{2}}
$$

and the angle between them is given by

$$
A f\left(2^{j}, x, y\right)=\arg \tan \left(\frac{W^{2} f\left(2^{j}, x, y\right)}{W^{1} f\left(2^{j}, x, y\right)}\right) .
$$

Thus. at each scale $2^{j}$, the modulus maxima of the wavelet transform are defined as point $(x, y)$ where the modulus image $\operatorname{Mf}\left(2^{j}, x, y\right)$ is locally maximum, along the gradient direct given by $A f\left(2^{j}, x, y\right)$. The line of position $(x, y)$ is normally the edge of the images.

\section{Application of Modulus Maxima in SpOT RECOGNITION}

Here, we develop the algorithm using modulus maxima to detect the edge of the microarray spots. Supposing the smoothing function is a simple 2-D, circular symmetric Gaussian function, $G$, which is given by

$$
G(x, y)=e^{-\left(x^{2}+y^{2}\right)} .
$$

Then. the two wavelets can be obtained by partial derivative of the Gaussian function at vertical and horizontal location

$$
\begin{aligned}
& \psi^{1}(x, y)=\frac{\partial}{\partial x} e^{-\left(x^{2}+y^{2}\right)}=-2 x e^{-\left(x^{2}+y^{2}\right)} \text { and } \\
& \psi^{2}(x, y)=\frac{\partial}{\partial y} e^{-\left(x^{2}+y^{2}\right)}=-2 y e^{-\left(x^{2}+y^{2}\right)} .
\end{aligned}
$$

Convolving these two wavelets with microarray function, the modulus maxima of the microarray image can be obtained by (4) and (5). The link of the location of the maxima is actually the edge, or contour, of the spot. Fig. 1 shows an example of a single microarray spot and the local maxima, contour, obtained from the algorithm. The local maxima detects not only the edge of the spot, but also a noise above the spot which is invisible to human eye and the intensity difference within the spot. However, what we wanted is just the edge of the spot. Within the edge, the intensity of the spot can be obtained as 14899 . The intensity of the original spot is 15604 . The error is just $4.5 \%$. Therefore, we can say this algorithm can accurately recognize the spot.

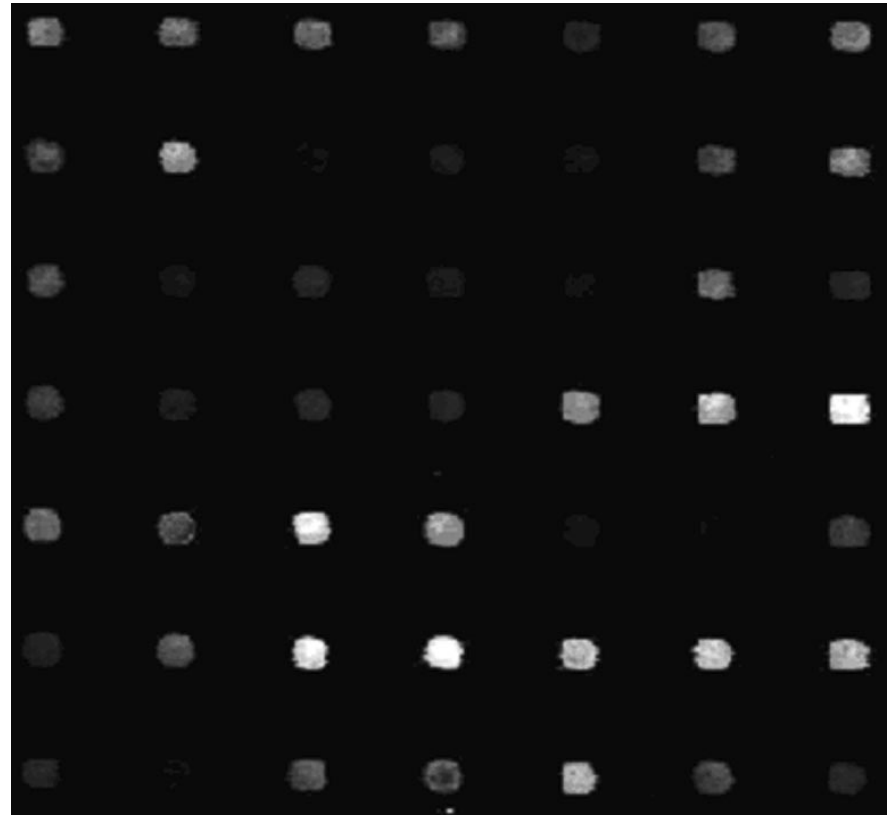

(a)

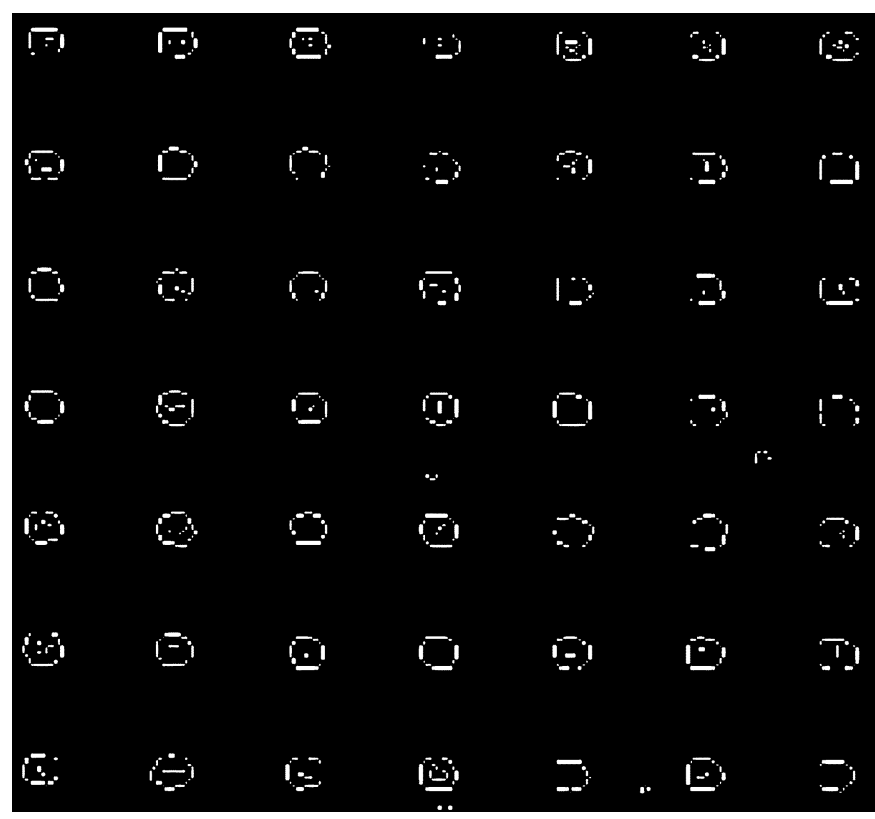

(b)

Fig. 2. Microarray image and its modulus maxima

Now we are applying this algorithm to a small microarray image with $7 \times 7$ array. Fig. 2(a) shows the original image. On this figure, some spots are very weak and difficult to be viewed by human eyes. After application of the modulus maxima detection algorithm, the edge of each spot can be identified, as shown in Fig. 2(b). That is the advantage of this algorithm, it will not leave even single weak spot.

As seen in Fig. 2, the shape of the spots varies from spot to spot. The distribution of the intensity within the spot is not uniform. Some weak areas can be identified by the local maxima within the spot. But when we do the intensity extraction, only the outer contour is concerned. Let us have a close examination of the spots, i.e., spots in column 4. Table I shows the original intensities and the relevant intensities within the contour together 
TABLE I

INTENSITIES OF COLUMN 4 SPOTS AS IN FIG. 2

\begin{tabular}{l|c|c|c}
\hline \multirow{2}{*}{ Spots } & \multicolumn{3}{|c}{ Intensities } \\
\cline { 2 - 4 } & Original & Within contour & Error(\%) \\
\hline 1 & 11526 & 10531 & 8.6 \\
\hline 2 & 2664 & 2425 & 8.97 \\
\hline 3 & 2712 & 2564 & 5.46 \\
\hline 4 & 4391 & 4055 & 7.65 \\
\hline 5 & 23219 & 22154 & 4.59 \\
\hline 6 & 37280 & 35832 & 3.88 \\
\hline 7 & 11511 & 11034 & 4.14 \\
\hline
\end{tabular}

with corresponding error values in that particular column. We can see only few pixel intensities are not included in the contour. These are sporadic pixels in the neighborhood of the spot.

\section{CONCLUSION}

From the above analysis, we can see using wavelet modulus maxima algorithm can precisely detect the contour of the microarray spots in spite of the shape of the spots. The algorithm is especially useful for those weak spots which are difficult to be detected. Thus, the intensities of the spot can be obtained with low error rate. The test result on example microarray image illustrates this is an effective approach to recognize the DNA spot.

\section{REFERENCES}

[1] M. B. Eisen. ScanAlyze. [Online]. Available: http://rana.stanford.edu/software

[2] GenePix 4000A User's Guide, 1999.

[3] QuantArray Analysis Software, Operator's Manual, 1999.

[4] S. Beucher and F. Meyer, "The morphological approach to segmentation: The watershed transformation," in Mathematical Morphology in Image Processing, Vol. 34 of Optical Engineering. New York, NY: Marcel Dekker, 1993, pp. 433-481.

[5] L. Vincent and P. Soille, "Watershed in digital space: An efficient algorithm based on immersion simulations," IEEE Trans. Pattern Anal. Machine Intell., vol. 13, pp. 583-598, June 1991.

[6] R. Adams and L. Bischof, "Seeded region growing," IEEE Trans. Pattern Anal. Machine Intell., vol. 16, pp. 641-647, June 1994.

[7] Y. H. Yang, M. J. Buckley, S. Dudoit, and T. P. Speed, "Comparison of methods for image analysis on cDNA microarray data," J. Comput. Graph. Stat., vol. 11, pp. 108-136, 2002.

[8] Y. Chen, E. R. Dougherty, and M. L. Bitterner, "Ratio-based decisions and the quantitative analysis of cDNA microarray images," J. Biomed. Opt., vol. 2, pp. 364-374, 1997.

[9] S. Mallat and W. L. Zhong, "Singularity detection and processing with wavelet," IEEE Trans. Inform. Theory, vol. 38, pp. 617-643, Mar. 1992.

X. H. Wang, photograph and biography not available at the time of publication.

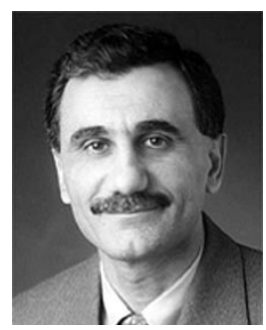

Robert S. H. Istepanian (M'91-SM'97) received the $\mathrm{Ph} . \mathrm{D}$. degree in electronic and electrical engineering from Loughborough University, Loughborough, U.K., in 1994.

From 1984 to 1998, he worked in different overseas industrial and academic positions. In 1988, he was a Visiting Research Fellow in the Department of Electronic and Electrical Engineering, Loughborough University. From 1994 to 1995, he was with the same department as a Postdoctoral Research Fellow. From 1996 to 1999, he was a Senior Lecturer at the University of Portsmouth, Portsmouth, U.K. From 1999 to 2000, he was an Associate Professor at Ryerson University, Toronto, ON, Canada, and also an Adjunct Professor with the University of Western Ontario, London, ON, Canada. $\mathrm{He}$ was also the Head of the Mobile Information Engineering and E-Med Systems research group in the Department of Electronic and Computer Engineering, Brunel University, London, U.K. He is currently Professor of Data Communications in the School of Computing and Information Systems and Director of the Mobile Information and Network Technologies Research Centre (MINT), Kingston University, London, U.K. He is Recipient, Investigator, and Coinvestigator of research grants from the Engineering and Physical Science Research Council, British Council, and Royal Society on intelligent computational systems applied to telesurgery systems. He is also a lead partner on several EU-funded projects on e-health. Other collaborative and funded projects from industry (Vodafone, Orange, and Motorola UK) in the areas of $\mathrm{m}$-health systems and microarray imaging technologies are also ongoing. He has published more than 120 refereed journal and conference papers, mostly in the areas of of wireless telemedicine and biomedical signal processing, and has edited three books in the areas of m-health, biomedical communications, and signal processing. His current research lies in the cognate areas of wireless communications and e-health and advanced coding theory for genomics and microarray imaging technologies.

Dr. Istepanian currently serves on the advisory board of IEEE TRANSACTIONS ON INFORMATION TECHNOLOGY IN BIOMEDICINE, and was one of the Founding Special Area Editors on mobile telemedicine. He was also the Guest Editor of the IEEE TRANSACTIONS ON INFORMATION TECHNOLOGY IN BIOMEDICINE Special Issue on Mobile Telemedicine Systems (Sept. 2000), and is Guest Editor of the forthcoming Special Issue on m-Health to be published in late 2003. He was the Co-Chair of the UK/RI chapter of the IEEE Engineering in Medicine and Biology Society (EMBS). He has also served on numerous technical committees, and has chaired and was an invited speaker for several national and international IEEE conferences, including the Telemed conferences of the Royal Society of Medicine, the Annual International Conference of the EMBS (EMBS'98 and '99), and the 2000 World Medical Congress. He was also the Technical Co-Chair of the 2003 IEEE EMBS Conference on Information Technology and Applications in Biomedicine (ITAB) and the Fifth International Workshop on Enterprise Networking and Computing in HealthCare (HealthCom'03). His biography is included in Who's Who in Science and Engineering (New Providence, NJ: Marquis, 2003).

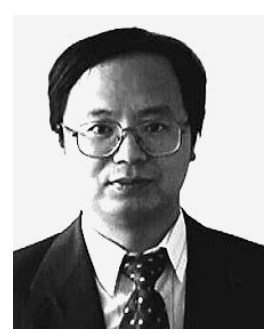

Yong Hua Song (M'90-SM'94) is currently a Professor of Network Systems at Brunel University, London, U.K., where he is Director of Brunel Network Systems Research Centre. His research interests include applications of advanced intelligent techniques and signal processing methods in various engineering problems. 